\title{
CRIPTOCOCOSE DO SISTEMA NERVOSO CENTRAL: AVALIAÇÃO DA TERAPEUTICA POR ANFOTERICINA-B, 5-FLUOROCITOSINA E MICONAZOLE EM 18 CASOS
}

\author{
J. P. S. NOBREGA* \\ J. A. LIVRAMENTO* \\ L. R. MACHADO** \\ A. SPINA-FRANCA***
}

O comprometimento do sistema nervoso central (SNC) em micoses oportunistas, especialmente na criptococose, constitui capítulo cada vez mais angustiante dentre as infecções do encéfalo e leptomeninges. Tal fato se deve à gravidade da doença em si, à inexistência até o momento de drogas desprovidas de efeitos colaterais importantes limitando sua utilização e, ainda, a um aumento na incidência. Decorre este último, em grande parte, do número crescente de pacientes submetidos a condutas terapêuticas que favorecem a transformação de agentes oportunisticos em agentes patogênicos ${ }^{3,5,7,13}$. Transplantados renais mantidos com imunossupressores e corticóides constituem o exemplo mais significativo quanto a esse aspecto.

Em 1975, Nobrega e col.9 chamaram a atenção para as limitações do uso da anfotericina-B (ANF-B), dada sua nefrotoxicidade, e relataram a utilização da associação desta droga com a 5-fluorocitosina (5-FC), de uso mais recente $1,4,6,8,10,11,14$.

O objetivo de presente trabalho é avaliar o resultado do tratamento de 18 casos de neurocripticocose e relatar a experiência inicial com o uso de nova droga, o miconazole, cuja eficácia no tratamento de algumas micoses entre elas a criptococose - tem sido registrada. Essa droga seria desprovida de efeitos nefrotóxicos e hepatotóxicos $2.1 \%$.

\section{CASUISTICA}

Foram estudados os casos de criptococose do SNC acompanhados na Clínica Neurológica do Hospital das Clínicas da Faculdade de Medicina da Universidade de São Paulo no período compreendido entre janeiro-1967 e julho-1978.

O número total de casos foi 18, tendo sido dois deles (casos 4 e 5) objeto de publicação anterior 9 A identificação dos pacientes, o tempo de internação, o ocorrência de óbitos e a concomitância de transplante renal, eståo relacionados na tabela 1. Constam dessa mesmo tabela a terapêutica adotada em cada caso e as complicações respectivas.

Trabalho do Centro de Investigações em Neurologia da Clínica Neurológica da Faculdade de Medicina da Universidade de São Paulo: *Médico-Assistente; *MédicoAdido; ***Professor-Adjunto. 


\section{COMENTARIOS}

Em apenas um dos 18 casos a sintomatologia era compativel a processo expansivo intracraniano. O diagnóstico de granuloma criptococócico foi estabelecido neurocirurgicamente nesse paciente (caso 1). Os 17 pacientes restantes apresentavam a sintomatologia clássica de meningoencefalite criptococócica, a maioria com hipertensão intracraniana severa.

Dos 18 pacientes, em 11 havia outra patologia associada: 7 eram transplantados renais; em 2 havia tuberculose associada (casos 6 e 9); em 1 havia referência a adenocarcinoma do colon do útero, submetido a radioterapia 5 anos antes (caso 11); em 1 foi diagnosticado diabetes melito durante a internação (caso 17).

Ocorreram 10 óbitos, em 9 diretamente relacionados à criptococose e, em um (caso 7), devido a tuberculose adquirida por ocasião de transplante renal e evidenciada posteriormente à cura da neurocriptococose.

A gravidade do quadro clínico em 5 pacientes (casos 2, 3, 6, 9 e 13) levou ao óbito antes de ser iniciado o tratamento ou nas respectivas fases iniciais. Neles, não foi possível avaliar a terapêutica adotada. Esta foi efetivamente realizada nos 13 pacientes restantes, nos quais pode ser devidamente avaliada. Entre esses pacientes ocorreu óbito diretamente relacionável à criptococose em 4 ( $\operatorname{casos} 4,10,15$ e 16); em 9, a resposta terapêutica foi satisfatória.

Foram utilizadas sistematicamente duas drogas: a ANF-B e a 5-FC, esta última a partir do caso 3. Só no caso 18 foi utilizada pela primeira vez o miconazole, após suspensão de ANF-B e 5-FC em decorrência de efeitos colaterais não controláveis.

A ANF-B foi empregada por via intravenosa (IV), intrarraqueana (IR) e intraventricular (V). As doses variaram de acordo com a via utilizada. Para a via IV foram utilizadas as doses convencionais para pacientes isentos de patologia renal e doses mais baixas em pacientes com nefropatia e anteriormente discutidas ${ }^{0}$. Variações nas doses foram estabelecidas ainda, caso a caso, na decorrência da maior ou menor tolerância individual à droga. Para a via IR sempre foi utilizada a punção lombar, iniciando-se o tratamento com $0,1 \mathrm{mg}$ por aplicação e aumentando a dose progressivamente, na dependência da tolerância do paciente, até atingir $1 \mathrm{mg}$ por aplicação, efetuada em dias alternados. Para a via V foi adotado critério semelhante. A aplicação por esta via foi feita mediante a introdução da droga em câmara de Rickham conectada a um dos ventrículos laterais.

A 5-FC foi utilizada por via oral, na dose de $200 \mathrm{mg} / \mathrm{kg}$ de peso/dia em pacientes sem comprometimento de função renal. $\mathrm{Na}$ presença deste, a dose diária foi baseada na depuração de creatinina.

O miconazole foi empregado por via IV e IR, na dose de $30 \mathrm{mg} / \mathrm{kg}$ de peso/dia para a via IV e de $20 \mathrm{mg}$ por aplicação para a via IR. Esta última foi empregada em dias alternados.

Em relação à ANF-B e ao miconazole, quaisquer que fossem as vias utilizadas, não foi estabelecido limite para a dose total, que variou de caso para caso. A 5-FC foi ainda utilizada por mais 6 meses após a alta hospitalar. 


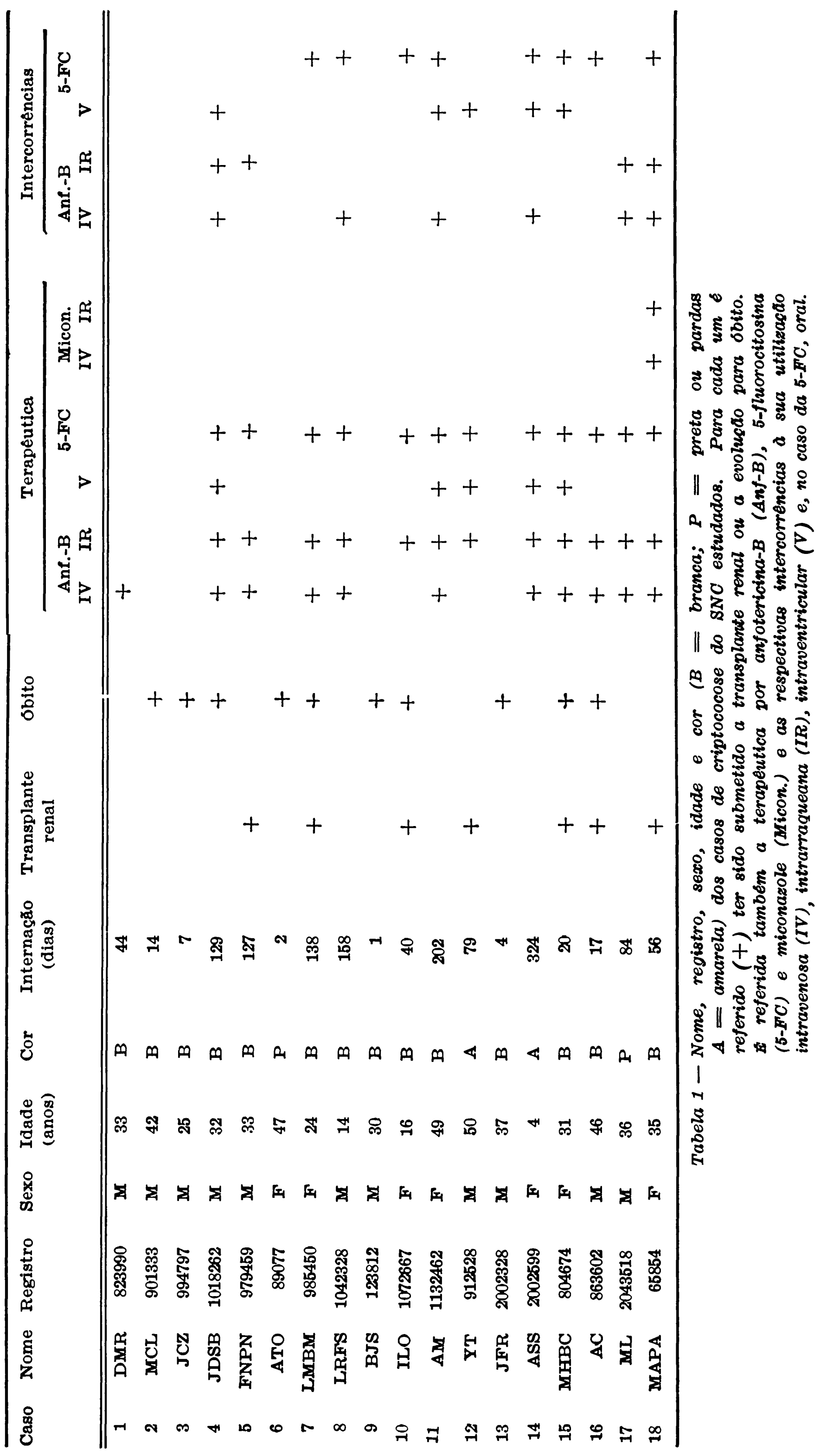


$\mathrm{Na}$ avaliação da cura e subsequente suspensão do tratamento foram adotados dois critérios: o do quadro clínico e o do quadro do LCR. Em geral, a melhora clínica antecedia de muito a melhora do LCR. Em três pacientes ( $\operatorname{casos} 11,14$ e 18) houve persistência do fungo no LCR, como única alteração, por período de tempo variável ( 7 meses no caso $11 ; 3$ meses no caso $14 ; 2$ meses no caso 18, este ainda em observação). Neles, as culturas do sedimento do LCR permaneceram estéreis e não ocorreram modificações ulteriores do quadro clínico ou da composição do LCR. Tal fato justificou a conduta de suspender o tratamento na vigência de culturas do sedimento do LCR estéreis para o fungo.

Dos 13 pacientes tratados, apenas um (caso 1) não apresentou intercorrências durante o tratamento.

Dos 11 pacientes tratados com ANF-B IV, houve intercorrências em 6: comprometimento da função renal em 5 pacientes (casos 4, 11, 14, 17 e 18); hipopotassemia em 1 (caso 8); insuficiência cardíaca congestiva em 1 (caso14). $E$ de notar que dos 11,5 eram transplantados renais, tendo havido intercorrências em apenas um deles (caso 18). Assim sendo, estes dados não justificam contraindicar a utilização da ANF-B por via sistêmica em transplantados renais, como é recomendado por vezes.

Dos 12 pacientes tratados com ANF-B IR, houve intercorrências em 4: paraparesia crural transitória em 3 (casos 5,17 e 18; hiperproteinorraquia em um (caso 4). Nesses casos foi suspenso definitivamente o tratamento por essa via.

Dos 5 pacientes tratados com ANF-B por via intraventricular, todos apresentaram intercorrências: meningite secundária em um caso (caso 4 ) e reações neurovegetativas severas nos demais (casos 11, 12, 14 e 15). A utilização da medicação por essa via foi suspensa a partir de observações no caso 16 , até haver melhor avaliação da respectiva eficácia.

Dos 12 pacientes tratados com $5-\mathrm{FC}$, houve intercorrências em 8 , as quais levaram a suspender definitivamente o emprego da droga. Essas intercorrências estavam relacionadas a discrasias sanguineas, principalmente. Foram observadas: anemia (casos 7, 8 e 14); pancitopenia (casos 10, 15, 16 e 18); leucopenia (caso 11) e alterações hepáticas (caso 16). Dos 12 pacientes, 7 eram transplantados renais, tendo havido intercorrências em 5 .

Apenas um paciente foi tratado com miconazole (caso 18), após suspensão do tratamento com ANF-B e 5-FC devida a efeitos colaterais indesejáveis surgidos tardiamente. Tratava-se de paciente transplantado renal e que suportou satisfatoriamente o emprego do miconazole em niveis terapêuticos.

A análise dos resultados obtidos permite recomendar, na terapêtutica para a criptococose do SNC, a associação da ANF-B, por via intravenosa e intrarraqueana, com a 5-FC, por via oral. Deve ser chamada a atenção, entretanto, para a dificuldade de uniformização do tratamento, devido às inúmeras intercorrências que podem ser observadas. Em sua maioria, elas decorrem da toxicidade das drogas utilizadas, o que motiva a pesquisa de novos medica- 
mentos, mais eficientes e menos tóxicos, e que tornem menos sombrio o prognóstico da neurocriptose. O miconazole foi introduzido nesse sentido. Todavia, é necessário reunir maior experiência antes de aceitar as possibilidades adequadas que lhe são apontadas.

\section{RESUMO}

São apresentados 18 casos de criptococose do sistema nervoso central e são tecidos comentários a propósito do tratamento e das dificuldades respectivas, especialmente em pacientes transplantados renais. É chamada a atenção para as eventuais possibilidades de nova droga introduzida para o tratamento da afecção e empregada em um dos casos relatados, o miconazole.

\section{SUMMARY}

Cryptococcosis of the central nervous system: therapeutical evaluation of amphotericin-B, 5-fluorocvtosine and miconazole in 18 cases.

Results obtained in the treatment of cryptococcosis of the central nervous system with amphotericin-B, 5-fluorocytosine and miconazole are evaluated. The evaluation is based upon 18 cases. Emphasis is given to data pertinent to aspects proper to 7 of them, submitted to chronic immunossupressive treatment due to previous kidney transplantation. Side effects varied from case to case in the patients submitted to amphotericin-B and 5-fluorocytosine treatment. The intrathecal and the intraventricular administration of amphotericin-B was followed by complications of several types. Miconazole was used in one case. Its intravenous and its intrathecal administration did not provoke remarkable side effects.

\section{REFERENCIAS}

1. BEINE, J. P.; LONTIE, M. \& VANDEPITTE, J. - Cryptococal meningoencephalitis and 5-fluorocytosine. Brit. med. J. 5753:107, 1971.

4. DERESINSKI, S. C.; LILLY, R. B.; LEVINE, H. B.; GALGIANI, J. N. \& STEVENS, D. A. - Treatment of fungal meningitis with miconazole. Arch. int. Med. $137: 1180,1977$.

3. EVERETT, B. A.; KUSSKE, J. A.; RUSH, J. L. \& PRIBRAM, H. W. - Cryptococcal infection of the central nervous system. Surg. Neurol. 9:157, 1978.

4. FASS, R. J. \& PERKINS, R. L. - 5-fluorocytosine in the treatment of cryptococcal and candida mycoses. Ann. int. Med. 74:535, 1971.

5. FETTER, B. F.; KIINTWORTH, G. K. \& HENDRY, W. S. - Mycoses of the Central Nervous System. Williams \& Wilkins Co., Baltimore, 1967.

6. GIORGI, D. R.; REIS, J. B.; PUPO, P. P. \& LIMA, J. G. C. - Tratamento da criptococose do sistema nervoso pela anfotericina-B. Arq. Neuro-Psiquiat. (Såo Paulo) 17:377, 1859. 
7. GIORGI, D. R.; REIS, J. B.; BEI, A. \& REIS FILHO, J. B. - Criptococose do sistema nernoso central. Arq. Neuro-Psiquiat. (São Paulo) 32:77, 1974.

8. MEDOFF, G.; KOBAYASHI, G. S.; KWAN, C. N.; SCHLESSINGER, D. \& VENKOV, P. - Potentiation of rifampicin and 5-fluorocytosine as antifungal antibiotics by amphotericin-B. Proc. nat. Acad. Sci. 69:196, 1972.

9. NOBREGA, J. P. S.; LIVRAMENTO, J. A. \& SPINA-FRANÇA, A. - 5-fluorocitosina e anfotericina-B no tratamento da criptococose do sistema nervoso central. Arq. Neuro-Psiquiat. (São Paulo) 33:210, 1975.

10. SPINA-FRANÇA, A. \& SILVA, J. B. - Diagnóstico e tratamento da criptococose do sistema nervoso central. Considerações sobre 16 casos. $\Lambda$ rq. Neuro-Psiquiat. (São Paulo) 26:115, 1968.

11. SPINA-FRANÇA, A. - Terapêutica das neuromicoses oportunisticas. In Lacaz, C. S. ed. - Infecções por Agentes Oportunistas. Ed. Univ. São Paulo, 1977 pág. 155.

12. SUNG, J. P.; GRENDAHL, J. G. \& LEVINE, H. B. - Intravenous and intrathecal miconazole therapy for systemic mycoses. West. J. Med. 126:5, 1977.

13. TOLOSA, A.; LACAZ, C. S. \& SPINA-FRANCA, A. - Criptococose do sistema nervoso central. Registro de um caso. Arq. Neuro-Psiquiat. (São Paulo) 14:171. 1956.

14. UTZ, J. P. - Current and future chemotherapy of central nervous system fungal infections. In Thompson, R. A. \& Green, J. R., eds. - Advances in Neurology 6:127. Raven Press, New York, 1974.

Centro de Investigaçes em Neurologia - Caixa Postal 5199 - 0t1000 8ão Paulo, SP - Brasil. 